\title{
Note on the paper, of Dr Bevan B. Baker, An Extension of Heaviside's Operational Method of Solving Differential Equations.*
}

By T. Kauckí.

(Received 3rd March 1925. Read 5th June 1925.)

$\S 1$. Let $f(x)$ and $F(x)$ be polynomials which are supposed to be decomposed into a series of $\boldsymbol{n}$ terms as

$$
\frac{f(x)}{F(x)}=\sum_{p=1}^{n} \frac{f_{\nu}(x)}{F_{\nu}(x)}
$$

Further let $\theta$ be a distributive operation and $\phi(x)$ be a given function. Then the functional equation

$$
F^{\prime}(\theta) y(x)=f(\theta) \phi(x)
$$

has a solution of the form

$$
y(x)=\sum_{y=1}^{n} y_{\nu}(x)
$$

where $y_{v}(x)(v=1,2, \ldots n)$ is the solution of the equation

$$
\begin{gathered}
F_{v}(\theta) y_{\nu}(x)=f_{\nu}(\theta) \phi(x) \dagger \\
(v=1,2, \ldots n) .
\end{gathered}
$$

The equation (2) may be written symbolically as follows

$$
\frac{f(\theta)}{F^{\prime}(\theta)} \phi(x)=\sum_{\nu=1}^{n} \frac{f_{\nu}(\theta)}{F_{\nu}(\theta)} \phi(x) ;
$$

and, by a special suitable choice of the decomposition (1) and of the functions $\phi, f, F$, this last formula $\left(2^{\prime}\right)$ leads to a series of results both in the differential and in the difference calculus.

- Proc. Bdinburgh Math. Soc., XLII (1924), 95-103.

† The demonstration of the equation (2), with certain results belonging to the caloulus of differences are given by the author in the paper, " $O$ redukol

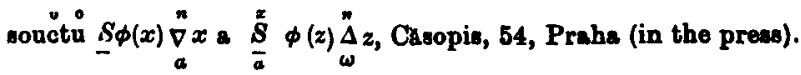




\section{6}

\$2. For example let the degree of $f(x)$ be less than the degree of $F(x)$, and suppose that the decomposition (1) is into partial fractions. In this case the relation (2) yields

$$
\frac{f(\theta)}{F(\theta)} \phi(x)=S \frac{\phi(x)}{\theta-r}\left[\frac{f(r)}{F(r)}\right],
$$

where $\frac{\phi(x)}{\theta-r}$ is the solution of the equation

$$
(\theta-r) y(x)=\phi(x),
$$

and $S$ means the sum of residues of the function

$$
\frac{\phi(x)}{\theta-r} \cdot \frac{f(r)}{F(r)}
$$

with regard to the poles of $F(r)$.

Further let

$$
D=\frac{d}{d x}
$$

and let us consider the solution of the equation

$$
(D-r) y(x)=\phi(x)
$$

for which $y(0)=0$. In this case

$$
y(x)=\int_{0}^{x} e^{r(x-z)} \phi(z) d z
$$

and formula (3) gives

$$
\frac{f(D)}{F(D)} \phi(x)=\int_{0}^{x} S e^{r(x-z)}\left[\frac{f(r)}{F(r)}\right] \phi(z) d z .
$$

To simplify matters let us suppose that $F(r)$ is of degree $n$ and has only simple zeros $r_{\nu}$. Then if we write

the equation (4) becomes

$$
A_{\nu}=\frac{f\left(r_{\nu}\right)}{F\left(r_{\nu}\right)}
$$

$$
\frac{f(D)}{F(D)} \phi(x)=\sum_{v=1}^{n} A_{\nu} \int_{0}^{x} e^{r_{\nu}(x-z)} \phi(z) d z .
$$

By proper choice of $\phi(x)$ in (5) the formulae of Bromwich, Carson, Heaviside and Baker may severally be deduced.

Brno (Czechoslovakia), February 1925. 